\title{
Thiamine deficiency and cerebrospinal fluid 5-hydroxyindoleacetic acid: a preliminary study
}

\author{
MI BOTEZ, ${ }^{*} \dagger$ SIMON N YOUNG, $\ddagger$ JOCELYNE BACHEVALIER, ${ }^{*} \dagger$ SERGE GAUTHIER§
}

From the Clinical Research Institute of Montreal, ${ }^{*}$ Department of Neurology, Hôtel-Dieu Hospital, $\dagger$ and Departments of Psychiatry $\ddagger$ and Neurology and Neurosurgery, $\S$ McGill University, Montreal, Quebec, Canada

SUMMARY In three out of five patients with low cerebrospinal fluid thiamine concentrations, the 5-hydroxyindoleacetic acid (5HIAA) values also were low. All patients received thiamine replacement therapy; they underwent a second lumbar puncture after 13, 6, 7, 5 and 45 days of treatment. In all patients blood and cerebrospinal fluid thiamine values rose after treatment. In those patients with initially low CSF 5HIAA, thiamine treatment increased 5HIAA markedly.

Thiamine deficiency is associated with an impairment of 5-hydroxytryptamine (5HT) neurons in experimental animals. ${ }^{1}$ In rats, a long-lasting folate deficiency can induce a secondary thiamine deficiency $^{2}$ and some of the behavioural changes in these animals can be reversed by administration of high doses of thiamine. ${ }^{3}$ Recently we have reported ${ }^{4}$ that folate deficient patients, who exhibit a folateresponsive neuropyschiatric syndrome have low levels of the 5HIAA in their cerebrospinal fluid (CSF). Low values of CSF 5-hydroxyindoleacetic acid (5HIAA) were also reported in patients with inborn errors of folate metabolism. ${ }^{5}$ We now wish to report on CSF 5HIAA levels in a small group of thiamine deficient patients before and after thiamine supplementation. To our knowledge, this is the first study on the correlations between thiamine and 5HIAA in the human CSF.

\section{Methods and Patients}

Five patients were studied. Blood and CSF were taken before and after thiamine replacement therapy, for the measurement of blood and CSF thiamine, serum and CSF folate, and CSF tryptophan and 5HIAA levels. Blood and CSF samples were collected at 9.00-9.30 a.m. The last dose of thiamine was taken 14 hours before the second

Address for reprint requests: Dr MI Botez, Clinical Research Institute of Montreal, 110, Pine Avenue West, Montreal, PO, Canada H2W 1 R7.

Received 22 November 1981 and in revised form 6 March 1982 Accepted 18 March 1982 lumbar puncture. The whole blood and CSF thiamine levels were assayed in duplicate, using Lactobacillus fermenti. ${ }^{6}$ When low values of thiamine were found, an additional incubation was carried out with added thiamine to check that no inhibitors of bacterial growth were present. ${ }^{7}$

The folate determinations (serum and CSF) were carried out using both Lactobacillus case $^{8}$ and radioisotope methods. ${ }^{9}$ Values for the two methods were in agreement and only the values for the radioisotope method are shown in the table. Tryptophan and 5HIAA were measured by high performance liquid chromatography with fluorometric detection. ${ }^{10}$ Informed consent was obtained in all patients for the second lumbar puncture.

Patients 1-4 (table) had alcohol-induced neurological disorders. Patients 1,3 and 4 had cerebellar ataxia and patient 2 suffered from alcoholic polyneuropathy. Patient 5 who abstained totally from alcohol had cerebellar ataxia resulting from 25 years of treatment for epilepsy with 300 $\mathrm{mg} /$ day of phenytoin. On admission to the hospital she had also anticonvulsant-induced dyskinesias. ${ }^{11}$ The first four patients received $300 \mathrm{mg} /$ day of thiamine orally for $13,6,7$ and 5 days respectively between the collection of the two CSF samples. The dietary habits were not modified during the thiamine replacement therapy. The fifth patient received $100 \mathrm{mg}$ of thiamine orally per day for 45 days. None of the patients were receiving thiamine before hospitalisation but patient 1 received $100 \mu \mathrm{g}$ of vitamin $B_{12}$ daily for two months before the first lumbar puncture; the same patient received $10 \mathrm{mg}$ of folic acid intramuscularly between the two CSF samples.

\section{Results}

The table shows that all five patients had low CSF thiamine values initially but that these values rose 
Table Laboratory findings

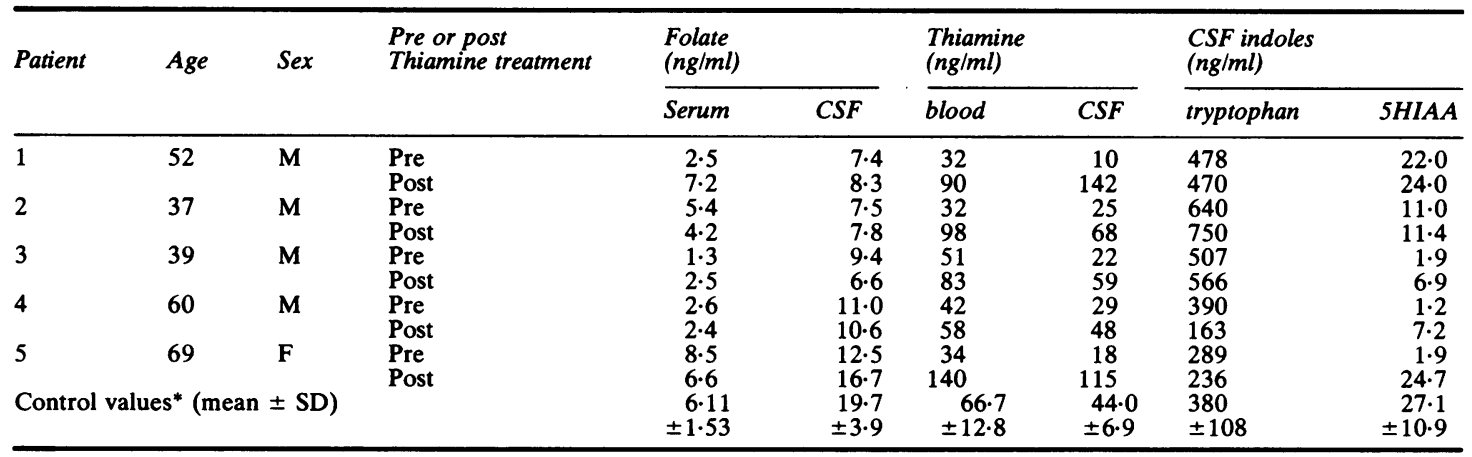

*Control values are the mean of 41 for the folate, 22 for the thiamine and 15 for the CSF indoles. Values for the controls were obtained from patients undergoing lumbar puncture for routine diagnostic purposes. They were on a normal diet and none of them were suffering from any clinical condition or were on any drugs known to affect folate, thiamine or indole metabolism.

after thiamine treatment. Patient 3 had a pretreatment blood thiamine in the normal range. However, blood levels are more dependent on variations in the diet and a true vitamin deficiency is sometimes seen only in the CSF.12 Patients 1-4 had some evidence of borderline folate deficiency but patient 5 was normal in this respect.

CSF tryptophan values were normal in all patients (table). The mean pretreatment CSF 5HIAA for the folate deficient patients $(7.6 \mathrm{ng} / \mathrm{ml})$ was less than control values ( $p<0.005$, two-tailed Student's $t$ test). Pretreatment CSF 5HIAA values were low (below mean-2 $\times$ SD of controls) in three out of five patients (nos 3-5). In these three patients thiamine treatment increased CSF 5HIAA markedly and the largest rise was seen in the patient no 5 who had been treated for the longest time with thiamine.

\section{Discussion}

The changes in CSF 5HIAA values appeared to result from thiamine treatment and not from some other nutritional factors because the dietary habits were not modified during the thiamine replacement therapy, and because the serum and CSF folate values (except case no 1), as well as the CSF tryptophan levels, did not differ significantly in the whole group before versus after thiamine therapy. Our data suggest that thiamine deficiency can decrease 5HT metabolism in human by an unknown mechanism that can be reversed by thiamine supplementation. However, two of the five patients had normal SHIAA values which were only increased minimally by thiamine supplementation. This suggests that thiamine deficiency alone does not necessarily impair brain 5HT metabolism in so far as this is indicated by CSF 5HIAA values.

The last point to be stressed is that patient no 5 who had been treated with phenytoin had low CSF 5HIAA values and low blood and CSF thiamine levels. In previous studies ${ }^{13}{ }^{14}$ we found abnormally low thiamine values in both whole blood and CSF in phenytoin-treated epileptics. One of us (MIB unpublished observations) studied recently two other cases of chronic PHT-induced cerebellar ataxia with low blood and CSF thiamine values. Therefore this study raises some important questions for future research namely: (1) to what extent is the phenytoin-induced chonic cerebellar ataxia ${ }^{15}$ correlated with a lowering of thiamine levels?; (2) what is the mechanism by which phenytoin usually lowers the levels of CSF 5HIAA ${ }^{16}$; is this mechanism correlated with folate deficiency, with thiamine deficiency or with both? Further studies are needed in both humans (epileptic and non-epileptic patients) and animals to verify to what extent the disturbed serotonin metabolism is correlated not only with folate values as has been already emphasise ${ }^{1617}$ but also with thiamine metabolism.

\section{References}

${ }^{1}$ Plaitakis A, Nicklas WJ, Berl S. Thiamine deficiency: Selective impairment of the cerebellar serotonergic system. Neurology (Minneap) 1978;28:691-8.

2 Thomson AD, Frank O, De Angelis B, Baker $H$. Thiamine depletion induced by dietary folate deficiency in rats. Nutr Rep Int 1972;6:107-10.

${ }^{3}$ Bachevalier J, Botez MI. Behavioral effects of folic acid deficiency and folate oversupplementation in postweanling rats. In: Botez MI, Reynolds EH, eds. Folic acid in neurology, psychiatry and internal medicine. New York: Raven Press, 1979:237-47.

${ }^{4}$ Botez MI, Young SN, Bachevalier J, Gauthier S. Folate deficiency and decreased brain 5HT synthesis in man and rat. Nature 1979;278:182-3.

${ }^{5}$ Singer HS, Butler I, Rotenberg S, Valle D, Freeman J. 
Interrelationships among serum folate, CSF folate, neurotransmitters, and neuropsychiatric symptoms. Neurology (Minneap) 1980;30:419 (Abs).

6 Baker H, Frank O. Clinical vitaminology. New York: Interscience Publishers, 1968:7-21.

${ }^{7}$ Bachevalier J, Joyal C, Botez MI. Blood thiamine and blood folate levels. Internat $J$ Vit Nutr Res 1981;51:205-10.

${ }^{8}$ Herbert V. Aseptic addition method for Lactobacillus casei assay of folate activity in human serum. J Clin Pathol 1966;19:12-16.

9 Waxman S. The value of measurement of folate levels by radioassay. In: Botez MI, Reynolds EH, eds. Folic acid in neurology, psychiatry and internal medicine. New York: Raven Press, 1979:47-53.

${ }^{10}$ Anderson GM, Purdy WC. A liquid chromatographicfluorometric system for the analysis of indoles in physiological samples. Anal Chem 1979;51:283-6.

${ }^{11}$ Chadwick D, Reynolds EH, Marsden CD. Anticonvulsant-induced dyskinesias: a comparison with dyskinesias induced by neuroleptics. J Neurol Neurosurg Psychiatry 1976;39:1210-8.

12 Reynolds EH. Cerebrospinal fluid folate: clinical studies.
In: Botez MI, Reynolds EH, eds. Folic acid and neurology, psychiatry and internal medicine. New York: Raven Press, 1979:195-203.

${ }^{13}$ Botez MI, Joyal C, Maag U, Bachevalier J. Low blood thiamine levels in phenytoin treated epileptics. A preliminary comparative study. Nutr Rep Int 1981;24:415-20.

${ }^{14}$ Botez MI, Joyal C, Maag U, Bachevalier J. Cerebrospinal fluid folate and blood thiamine concentrations in phenytoin-treated epileptics. Canad J Neurol Sci (in press).

${ }^{15}$ Ghatak NR, Santoso RA, McKinney WM. Cerebellar degeneration following long-term phenytoin therapy. Neurology (Minneap) 1976;27:818-20.

${ }^{16}$ Young SN, Gauthier S, Anderson GM, Purdy WC. Tryptophan, 5-hydroxyindoleacetic acid and indoleacetic acid in human cerebrospinal fluid: interrelationships and the influence of age, sex, epilepsy and anticonvulsant drugs. J Neurol Neurosurg Psychiatry 1980;43:438-55.

${ }^{17}$ Reynolds EH, Chadwick D, Jenner P, Chanarin I. Folate and monoamine metabolism in epilepsy. J Neurol Sci 1975;26:605-11. 